\title{
Évaluation de la diversité et estimation de la biomasse des arbres d'alignement des communes du Plateau et de Cocody (Abidjan - Côte d'Ivoire)
}

\author{
Yao Jean Clovis KOUADIO ${ }^{*}$; Bi Tra Aimé VROH ${ }^{1}$; Zoro Bertin GONE BI ${ }^{1}$; Constant Yves ADOU \\ YAO1, 2 ; et Kouakou Edouard N'GUESSAN1 \\ ${ }^{1}$ Laboratoire de Botanique, UFR Biosciences, Université Félix Houphouët-Boigny, 22 BP 582 Abidjan 22, Côte \\ d'Ivoire. \\ ${ }^{2}$ Centre Suisse de Recherche Scientifique en Côte d'Ivoire, 01 BP 1301 Abidjan 01 \\ *Auteur pour correspondance : jeanclovis08@gmail.com
}

Original submitted in on $3^{\text {rd }}$ December 2015. Published online at www.m.elewa.org on 31st January 2016

http://dx.doi.org/10.4314/jab.v97i1.1

\section{RÉSUMÉ}

Objectif : La présente étude réalisée sur les arbres d'alignement des communes du Plateau et de Cocody a pour but d'évaluer la diversité des arbres d'alignement et leur rôle dans l'amélioration de l'environnement urbain.

Méthodologie et résultats : La méthode de relevés itinérants a permis de recenser des arbres plantés en alignement le long de 13 avenues et boulevards. Au cours de l'inventaire, les circonférences et les hauteurs des individus de $\mathrm{dbh} \geq 5 \mathrm{~cm}$ ont été notées. Nous avons par la suite évalué la biomasse des individus à partir d'équations allométriques génériques des arbres urbains. Les analyses ont permis de dénombrer 705 individus dont 565 au Plateau et 140 à Cocody. Ces individus sont repartis entre 29 espèces, 20 genres et 17 familles. Les espèces les plus représentées sont Albizia lebbeck, Hevea brasiliensis, et Terminalia mantaly. On note $51,72 \%$ d'espèces de la région Guinéo-Congolaise et $41,37 \%$ d'espèces exotiques introduites. La biomasse sèche des arbres des deux communes s'élève à 414,21 t/ha ; ce qui correspond à un stock de carbone de 207,105 t/ha.

Conclusions et application des résultats: Les résultats obtenus renseignent sur la diversité et le rôle compensatoire des arbres urbains dans la séquestration du dioxyde de carbone issu des activités anthropiques. Ces informations tirées des conclusions de la présente étude constituent une importante argumentation pour le maintien des arbres d'alignement dans la ville d'Abidjan.

Mots clés : biodiversité urbaine, espace vert urbain, Abidjan, Côte d'Ivoire 
Kouadio et al. J. Appl. Biosci. 2016 Évaluation de la diversité et estimation de la biomasse des arbres d'alignement des communes du Plateau et de Cocody (Abidjan - Côte d'Ivoire

Evaluation of diversity and estimate roadside trees biomass in the districts of Plateau and Cocody (Abidjan - Côte d'Ivoire)

\section{ABSTRACT}

Objective: This study on roadside trees common in the Plateau and Cocody aims to assess the diversity of roadside trees and their role in improving the urban environment.

Methodology and Results: The method of itinerant readings allowed to identify trees planted in alignment along 13 avenues and boulevards. During the inventory, the circumferences and heights of individuals of trees whose dhh $\geq 5 \mathrm{~cm}$ dbh were recorded. We subsequently assessed the biomass of individuals from generic allometric equations of urban trees. The analyzes allowed to count 705 individuals including 565 trees to Plateau and 140 in Cocody. These individuals are distributed among 29 species, 20 genera and 17 families. The most represented species are Albizia lebbeck, Hevea brasiliensis, Terminalia mantaly. $51.72 \%$ of species is noted in the Guineo-Congolese region and $41.37 \%$ of introduced exotic species. The dry biomass of trees on both districts amounted to $414.21 \mathrm{t} / \mathrm{ha}$; which corresponds to a carbon stock of $207.105 \mathrm{t} / \mathrm{ha}$.

Conclusions and application of the results: the results provide information on the diversity and compensatory role of urban trees in the sequestering carbon dioxide from human activities. The informations from the findings of this study are an important argument for the maintenance of street trees in the city of Abidjan.

Keywords: urban biodiversity, urban green space, Abidjan, Côte d'Ivoire

\section{INTRODUCTION}

Les villes abritent de nos jours, un nombre relativement élevé de formations végétales qui sont soit plantées ou naturelles. Ces formations végétales allant de quelques plantes isolées à des formations beaucoup plus vaste (Selmi, 2011; Mehdi et al., 2012), sont représentées par les parcs, les jardins publics, les jardins privés, les arbres d'alignement, les espaces délaissés etc. Elles sont désignés dans la littérature sous le vocable de forêts urbaines (Nowak, 1994 ; Nowak et al., 2006) ou encore d'espaces verts urbains (Jo, 2002). La plupart des travaux scientifiques reconnaissent en elles de nombreux bienfaits (Arrif et al., 2011) classés sous le thème de services écosystémique (Millenium Ecosystem Assessment, 2005). Ces services écosystémiques sont entre autres la réduction des polluants atmosphériques (Nowak et al., 2006), la réduction des îlots de chaleur (Akbari et al., 2001), l'approvisionnement en ressources (Lessard \& Boulfroy, 2008), la réduction du taux de dioxyde de carbone par le phénomène de la séquestration de carbone (Nowak \& Crane, 2002, Vroh et al., 2014). Ainsi, ces services écosystémiques rendus par les arbres, améliorent la qualité environnementale des villes dont dépend la santé des citadins (Jim \& Chen, 2008; Escobedo et al., 2010; Young, 2010). Cependant, l'arbre en ville fait l'objet de nombreuses pressions du fait de la démographie galopante, de l'extension ou de la construction des routes. En effet, plus de la moitié de la population mondiale vit en milieu urbain (Veron, 2007). Cette forte démographie, plus accentuée dans les grandes agglomérations a nécessité la création de plusieurs autres logements et routes au dépend des espaces verts. La conséquence directe de ce phénomène est la détérioration marquée des ressources arborées à travers l'étalement de la ville sur les espaces verts (Scheneiders et al., 2012). Le phénomène est plus remarquable dans les pays en développement, notamment en Afrique de l'ouest où la sensibilité des citadins à la présence des végétaux se révèle plus faible au fur et à mesure que la ville est plus densément construite (Rusterholz, 2003). Cette situation préjudiciable à l'environnement (Wolff, 2005) s'observe également en Côte d'lvoire et particulièrement dans la ville d'Abidjan. En effet, dans la ville d'Abidjan, les espaces verts, en général, et les arbres d'alignement, en particulier, 


\section{Kouadio et al. J. Appl. Biosci. 2016 Évaluation de la diversité et estimation de la biomasse des arbres}

d'alignement des communes du Plateau et de Cocody (Abidjan - Côte d'Ivoire

sont soumis à d'intenses pressions anthropiques. Les raisons qui fondent cet état de chose sont liées à la mise en place des aménagements, à l'extension et à la construction de routes. Ces raisons s'expliquent à travers les opérations de restructuration de la ville, la recherche d'espace et l'incivisme (Akionla, 2012). Ainsi, très souvent, les arbres d'alignement sont éliminés dès que leur présence gêne, sans même se soucier de leur rôle écologique pour l'être humain. Ce phénomène a débuté à Abidjan depuis les années 1980 par la suppression d'un terre-plein axial qui comportait une double rangée d'arbres vieux d'un quart de siècle lors de l'élargissement du Boulevard Valery Giscard d'Estaing (Haeringer, 1980). II a engendré la disparition des arbres d'alignement des voiries dans la plupart des communes d'Abidjan. Seuls quelques vestiges existent encore dans les communes du Plateau et de Cocody. Un tel phénomène suscite des inquiétudes relatives aux effets potentiels sur la conservation de la

\section{MATÉRIEL ET MÉTHODES}

Zone d'étude: Situées dans la ville d'Abidjan, les communes du Plateau et de Cocody qui représentent les zones d'étude s'étendent respectivement sur 2,5 $\mathrm{km}^{2}$ (Vroh et al., 2014) et $132 \mathrm{~km}^{2}$ de superficie (Figure 1).Le climat de la ville d'Abidjan comme décrit par Eldin (1971) est caractérisé par quatre saisons. II s'agit d'un climat de type équatorial. Selon Mangenot (1955), la végétation originelle est de type forêt dense humide caractérisée par Turraeanthus africanus (Welw. ex DC) Pellegr. (Meliaceae) et Heisteria parvifolia Sm. (Olacaceae). La commune du Plateau est considérée comme le " quartier des affaires » et la commune de Cocody, comme le quartier résidentiel de la ville d'Abidjan. Ces deux communes abritent plusieurs espaces verts dont les arbres d'alignement. Ces arbres d'alignement se rencontrent le long des grands biodiversité et de la qualité du cadre de vie humaine (Miller, 2005; Dye, 2008). Dans un tel contexte, l'on est amené à s'interroger sur la durabilité des arbres d'alignement dans ces deux communes connues d'Abidjan. La présente étude a été initiée dans le but de développer des arguments solides quant au maintien des arbres d'alignement et des autres types d'espace verts. Le principal défi de l'étude consiste donc à s'assurer que l'extension des voies et la construction de nouvelles routes ne constitueront plus une entrave à la conservation des arbres d'alignement dans la ville d'Abidjan. . L'objectif visé est d'évaluer la diversité des arbres d'alignement et leur rôle dans l'amélioration de l'environnement urbain. De façon spécifique, il s'est agi de déterminer la diversité des arbres plantés en alignement, d'estimer leur biomasse totale afin d'en déduire leur potentiel de stockage de carbone.

boulevards et avenues qui représentent nos différents sites d'étude. Dans cette étude, ce sont au total 11 boulevards et avenues pour la commune du Plateau et deux (2) pour la commune de Cocody qui ont été inventoriés (Figure 1). Dans la commune du Plateau nous avons les boulevards Angoulevant $(1,32 \mathrm{~km})$, Carde $(0,92 \mathrm{~km})$, Clozel $(1,63 \mathrm{~km})$, Republique $(1,69$ $\mathrm{km})$, Roume $(0,72 \mathrm{~km})$ et les avenues Chardy $(0,39$ $\mathrm{km})$, Delafosse $(1,32 \mathrm{~km})$, Dr Jamot $(0,48 \mathrm{~km})$, Franchet Desperet $(0,64 \mathrm{~km})$, Marchand $(0,65 \mathrm{~km})$, Terrasson de Fougère $(0,46 \mathrm{~km})$. Au niveau de la commune de Cocody, ce sont le Boulevard des Martyrs $(0,90 \mathrm{~km})$ et l'avenue $D_{33}(0,13 \mathrm{~km})$. La largeur de chacun des boulevards est de $15 \mathrm{~m}$ alors que celle d'une avenue est de $7 \mathrm{~m}$. 


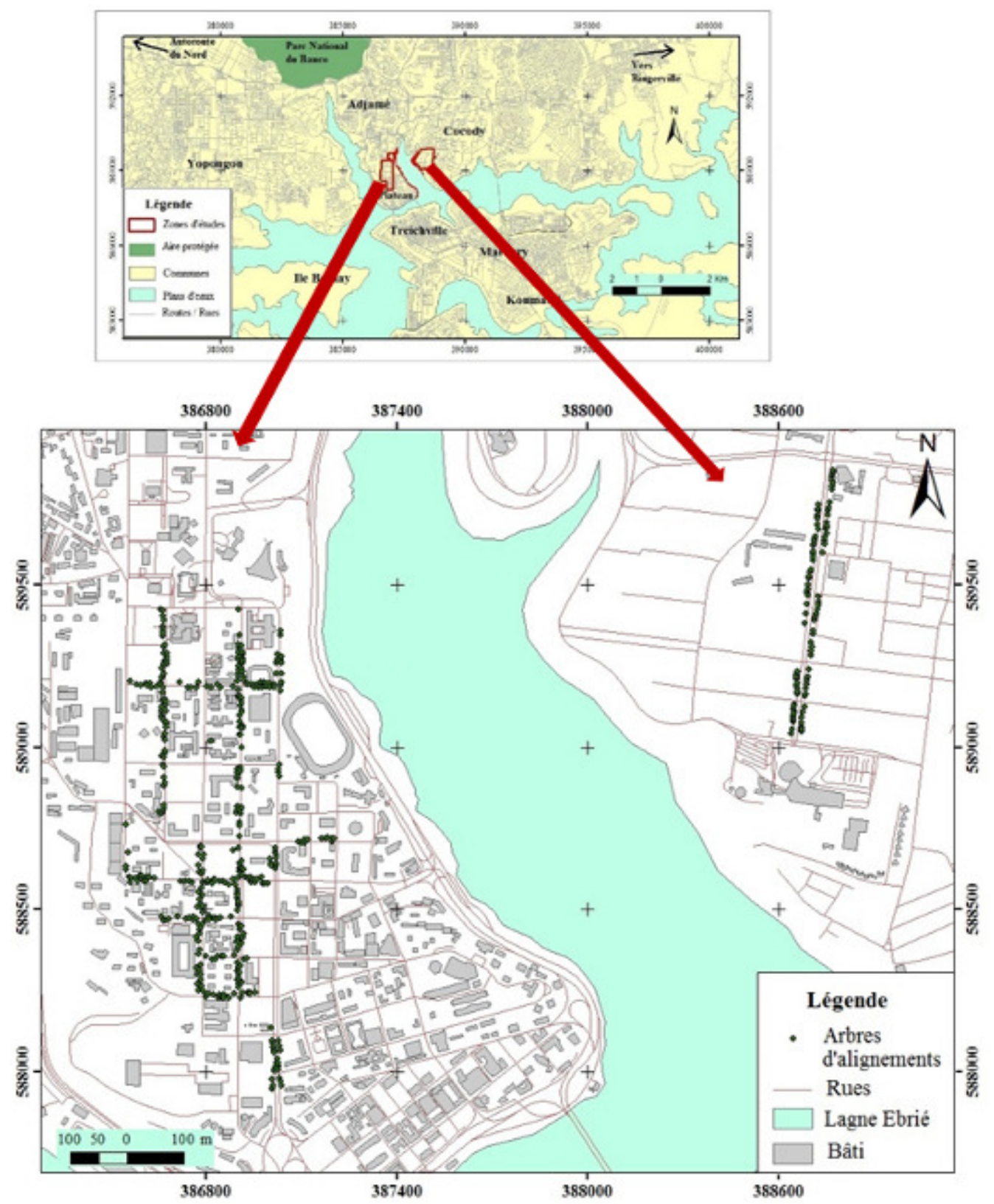

Figure 1 : Localisation des arbres d'alignement sur les différents sites d'étude

Collecte des données: La méthode de relevés itinérants a été utilisée pour la collecte des données floristiques. Elle a consisté à noter toutes les espèces rencontrées lors du parcours des différents boulevards et avenues. A cet effet, tous les arbres plantés en alignement aux abords des différentes voies ont été identifiées et leurs hauteurs estimées à l'aide de jalons emboités. Tous les individus ligneux ayant à $1,30 \mathrm{~m}$ de hauteur ou à hauteur de poitrine $(\mathrm{DBH})$ une tige d'un diamètre supérieur ou égal à $5 \mathrm{~cm}$ ont également été notés. Des échantillons des espèces non identifiés sur le terrain ont été récoltés et comparés à ceux de l'herbier du Centre National de Floristique (CNF) de I'Université Félix Houphouët Boigny en vue de leur identification. Pour la majorité des espèces exotiques ornementales, l'identification a été faite à partir des travaux de Aké-Assi (2002).

Analyse des données: L'analyse de la diversité floristique qualitative a porté sur les listes floristiques avec des informations sur le genre, la famille, les types 


\section{Kouadio et al. J. Appl. Biosci. 2016 Évaluation de la diversité et estimation de la biomasse des arbres}

d'alignement des communes du Plateau et de Cocody (Abidjan - Côte d'Ivoire

biologiques et chorologiques pour chacune des espèces. Les types biologiques et affinités chorologiques ont été déterminés, par comparaison à ceux énumérés par Aké-Assi $(2001 ; 2002$;). Les résultats sont par la suite donnés en pourcentage d'espèces, famille, genre, types biologiques et chorologiques. La densité (d) qui est le nombre d'individus (n) par unité de surface (s) a été calculée pour chaque type de voie (Avenue ou boulevard) grâce à la formule mathématique ci-dessous :

$$
d=\frac{n}{s}
$$

Pour estimer l'aire basale $(S)$, nous avons utilisé le diamètre (D) des arbres selon la formule mathématique

$$
S=\frac{\pi D^{2}}{4}
$$

La biomasse a été estimée à partir de modèles allométriques en utilisant les paramètres dendrométriques que sont le diamètre à hauteur de poitrine (DBH) et la hauteur (Brown et al., 1989; Brown, 1997) des arbres. En raison des lacunes en matière d'équations allométriques spécifiques relatives à la biomasse des arbres ou des arbustes dans les zones urbaines (Nowak et al., 1996 ; Jo, 2002), nous avons, pour cette étude, appliqué les équations allométriques générales des arbres urbains ou Urban General Equations (Aguaron \& McPherson, 2012) qui calculent la biomasse totale. Selon le type d'espèce (arbres urbains, palmier), trois équations allométriques spécifiques ont été utilisées. Ces différentes équations s'écrivent comme ci-après.

\section{RÉSULTATS}

Richesse et composition floristique: L'inventaire floristique a permis de recenser 705 arbres répartis entre 29 espèces, 20 genres et 17 familles (Tableau 1). Les nombre d'individus, d'espèces, de genres et de familles sont plus élevés sur les Boulevards que sur les Avenues. La commune du plateau compte 565 individus contre 140 dans la commune de Cocody. Dans les deux types d'espace (Avenues et Boulevards) des deux communes, les espèces comptant le plus d'individus sont au nombre de 4. II s'agit par ordre d'importance d'Albizia lebbeck (194 individus) dont $29,38 \%$ des individus sont sur les avenues et $70,10 \%$ sur les boulevards; Hevea brasilensis representé par 64 individus avec 10,76\% de ceux-ci sur les avenues contre $89,23 \%$ sur les boulevards. Par la suite, nous avons Terminalia mantaly représenté par 56 individus
Biom $_{\mathrm{t}}$ (arbres urbains $)=0,16155 \times \mathrm{DBH}^{2,310647}$

Biom $_{\mathrm{t}}($ palmiers $)=1,282 \times(7,7 \mathrm{H}+4,5)$

Dans ces formules, Biom $t$ désigne la biomasse totale exprimée en $\mathrm{kg} /$ tige et $\mathrm{H}$, la hauteur totale de l'arbre mesurée en mètre ; $\mathrm{DBH}$ désigne le diamètre à hauteur de poitrine exprimé en $\mathrm{cm}$. Enfin, la biomasse totale multipliée par 0,5 représente le stock de carbone correspondant (Brown \& Lugo, 1992 ; Malhi et al., 2004 ; GIEC, 2006). Aussi, l'équivalent carbone est obtenu en multipliant ce stock de carbone par 0,27. Vu la différence des espèces le long des voies, il se pourrait que leur planting ait tenu compte de certains paramètres tels que la largeur et la longueur de la voie, la commune d'échantillonnage (Plateau ou Cocody) et le type de voie (Avenue ou Boulevard). Nous avons vérifié ces éventuelles relations entre les espèces et les différentes variables qui pourraient influencer leur choix, à travers une analyse factorielle multiple (AFM). Les variables qualitatives sont la commune d'échantillonnage, le type de voie. Les variables quantitatives sont le nombre d'individus pour une espèce donnée, la largeur et la longueur de la voie.

Pour les paramètres densité, aire basale, biomasse, nous avons effectué des tests non paramétriques de Mann-Whitney, car nous étions en présence de données de variances inégales et non normalisées. Le niveau de significativité choisi pour ces analyses est de $5 \%(P=0,05)$. Le logiciel $\mathrm{R}$ a été utilisé pour la réalisation de tous ces tests statistiques.

tous présents sur les boulevards. Enfin, l'espèce Peltophorum pterocarpum (50 individus) se retrouve uniquement sur les avenues. Ces 4 espèces représentent $64 \%$ des individus de l'ensemble des espèces recensées. On rencontre Albizia lebbeck et Hevea brasiliensis uniquement au Plateau. Terminalia mantaly et Peltophorum pterocarpum ne se rencontrent que dans la commune de Cocody. Les familles les plus représentatives en nombre d'espèces sont celle des Moraceae $(29,41 \%$.) et des Caesalpiniaceae $(11,76$ $\%$.). Les Combretaceae, les Polypodiaceae et les Euphorbiacae comptent chacune 5,88\% des espèces. Au niveau des types biologiques, dans l'ensemble des espaces inventoriés, les microphanérophytes et les épiphytes sont les plus nombreux et partagent le même nombre d'espèce (11). Les épiphytes strictes sont 


\section{Kouadio et al. J. Appl. Biosci. 2016 Évaluation de la diversité et estimation de la biomasse des arbres d'alignement des communes du Plateau et de Cocody (Abidjan - Côte d'Ivoire}

moins nombreux que les épiphytes accidentels (Tableau 1).C'est sur les boulevards qu'on rencontre la majorité de ces espèces. Du point de vue chorologique, il a été relevé 12 espèces de la région GuinéoCongolaise (GC) et 12 espèces exotiques (i) ; ce qui représente les plus fortes valeurs (Tableau 1). Ces espèces de la région Guinéo-Congolaise se rencontrent uniquement sur les boulevards. C'est sur le boulevard Clozel de la commune du Plateau qu'on en rencontre en plus grand nombre. Les espèces les moins représentées sont les espèces de transition forêt-savanes (GC-SZ). Seulement 3 espèces de cette région phytogéographique ont été rencontrées. Toutes les (trois) 3 sont représentées sur les boulevards alors que seulement une (1) est présente sur les avenues.

Tableau 1: Récapitulatif de la richesse et de la composition floristique des différents types de voirie de Cocody et Plateau

\begin{tabular}{l|c|c|c}
\hline & Avenue & Boulevard & Total \\
\hline Nombre d'individus & 189 & 516 & 705 \\
\hline Nombre d'individus à $\mathrm{DBH} \geq 5 \mathrm{~cm}$ & 162 & 374 & 536 \\
\hline Nombre d'espèces & 11 & 28 & 29 \\
\hline Nombre de genres & 11 & 19 & 20 \\
\hline Nombre de familles & 10 & 16 & 17 \\
\hline Nombre de Microphanérophytes & 5 & 10 & 11 \\
\hline Nombre de Mésophanérophytes & 2 & 4 & 3 \\
\hline Nombre d'épiphytes strictes (Ep) & 3 & 3 & 8 \\
\hline Nombre d'épiphytes accidentels mp & 1 & 8 & 15 \\
\hline Nombre de GC & 4 & 15 & 3 \\
\hline Nombre de GC-SZ & 1 & 3 & 12 \\
\hline Nombre de i & 6 & 11 & \\
\hline
\end{tabular}

Biomasse totale et stock de carbone des arbres : La densité moyenne calculée s'élève à 130,76 $\pm 147,95$ Tige/ha (Tableau 2) sur les avenues contre 37,35 \pm 20,70 Tige/ha au niveau des boulevards. La différence observée entre ces valeurs est significative $\left(X^{2}=37 ; P=\right.$ $0,027)$. Ainsi, Les avenues sont plus denses que les boulevards. De même, l'aire basale reste plus élevée sur les avenues que sur les boulevards même si la différence statistique entre les deux valeurs n'est pas significative $\left(X^{2}=32 ; P=0,13\right)$. La biomasse totale des arbres d'alignement dans les deux communes de la ville d'Abidjan est de 562,33 $\pm 819,39$ tha sur les avenues. Sur les boulevards la valeur est plus faible. Ces biomasses converties en stock de carbone donnent 281,16 $\pm 409,7$ tha pour les avenue contre $133,05 \pm 181,7400$ t/ha sur les boulevards. Les variations des valeurs d'équivalent $\mathrm{CO}_{2}$ par type d'espace suivent les mêmes tendances observées pour les biomasses totales (Tableau 2). Le test de MannWhitney indique que les différences observées entre les valeurs de ces variables ne sont pas significatives (Tableau 2).
Paramètres ayant guidés la plantation des arbres : Les deux premiers axes de l'AFM restituent $70 \%$ de la variance des relations entre la flore et les caractéristiques des voies (Figure 2). L'examen du premier plan factoriel des variables met en évidence un axe 1 qui oppose les boulevards et les avenues. Cet axe décrit du côté positif les boulevards qui disposent des longueurs et des largeurs plus élevées. Dans sa partie négative, nous avons les avenues dont les longueurs et largeurs sont moindres. L'axe 2 oppose les deux communes. Chaque commune est caractérisée par un groupe d'espèce selon qu'on se présente sur un boulevard ou une avenue. Ainsi, les avenues de Cocody sont caractérisées par l'espèce Peltophorum pterocarpum qui est la plus fréquente alors que les avenues du Plateau comporte pour les plus fréquentes, les espèces telles que Eugenia malaccensis et Lagerstroemia speciosa. Aussi, les espèces caractéristiques des boulevards du Plateau diffèrent-elles de celles de Cocody. 
Kouadio et al. J. Appl. Biosci. 2016 Évaluation de la diversité et estimation de la biomasse des arbres d'alignement des communes du Plateau et de Cocody (Abidjan - Côte d'Ivoire

Tableau 2 : Paramètres structuraux et biomasse aérienne dans les différents espaces

\begin{tabular}{c|c|c|c|c}
\hline & & & \multicolumn{2}{|c}{ Statistique } \\
\hline Variables & Type de voie & Moyenne \pm Ecart type & $X^{2}$ & $P$ \\
\hline \multirow{2}{*}{ Densité $($ Tige/ha) } & Avenue & $130,76 \pm 147,95^{\mathrm{a}}$ & & \\
\cline { 2 - 3 } & Boulevard & $37,35 \pm 20,70^{\mathrm{b}}$ & 37 & 0,027 \\
\hline \multirow{2}{*}{ Aire basale $\left(\mathrm{m}^{2} / \mathrm{ha}\right)$} & Avenue & $39,06 \pm 32,5700$ & & \\
& Boulevard & $17,42 \pm 11,9500$ & 32 & 0,13 \\
\hline \multirow{2}{*}{ Biom $_{\mathrm{t}}(\mathrm{t} / \mathrm{ha})$} & Avenue & $562,33 \pm 819,39$ & \multirow{2}{*}{27} & 0,43 \\
\cline { 2 - 3 } & Boulevard & $266,09 \pm 363,4800$ & 27 & \\
\hline
\end{tabular}
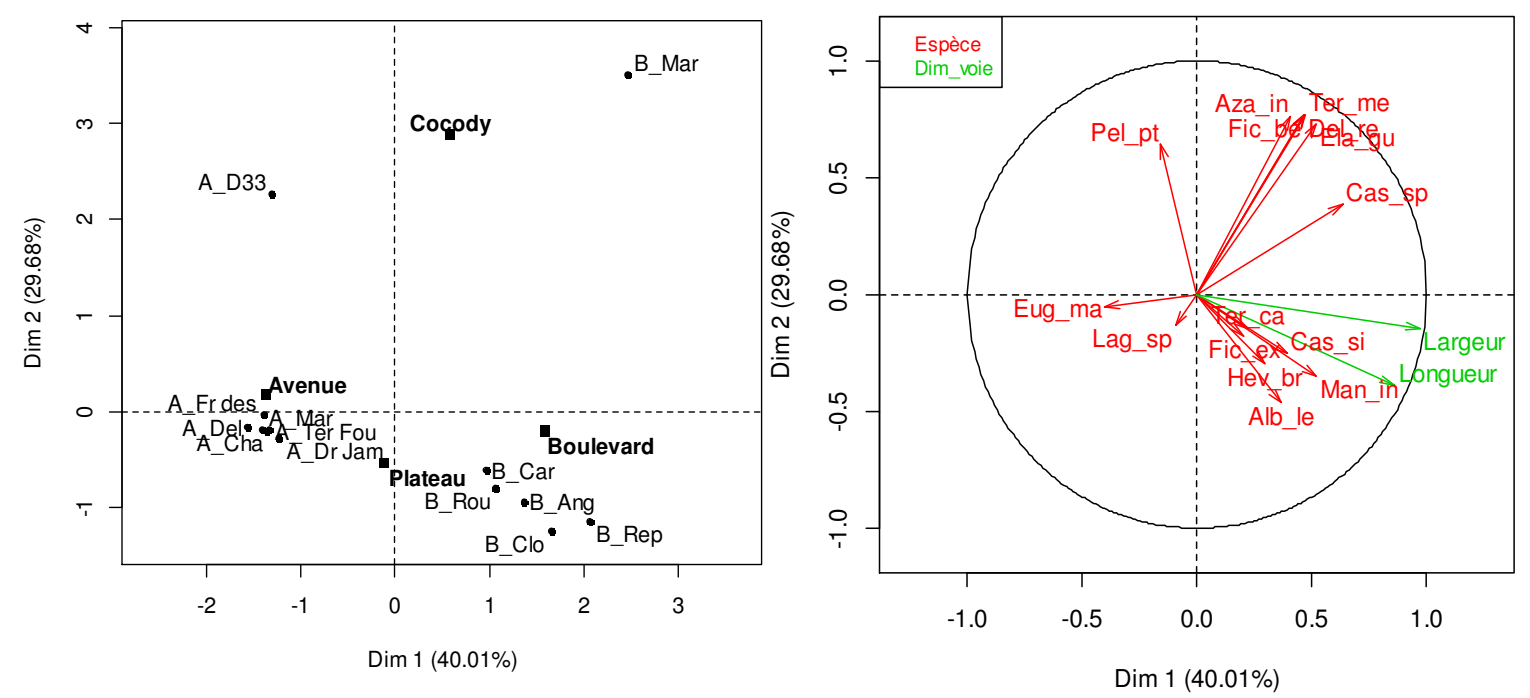

Figure 2 : Carte factorielle et Cercle de corrélation des groupes de variables utilisés dans l'AFM

Légende: A_Cha: Avenue Chardy ; A_D33: Avenue D33; A_Del: Avenue Delafosse ; A_Dr Jam : Avenue Dr Jamot ; A_Fr des: Avenue Franchet Desperet; A_Mar: Avenue Marchand; A_Ter Fou : Avenue Terrasson de Fougère ; B_Ang : Boulevard Angoulevant ; B_Car : Bpulevard carde ; B_Clo : Boulevard Clozel ; B_Mar : Boulevard Marchand ; B_Rep : Boulevard de la Republique ; B_Rou: Boulevard Roume; Alb_le: Albizia lebbeck; Aza_in : Azadirachta indica; Cas_si: Cassia siamea; Cas_sp: cassia sp; Del_re: Delonix regia; Ela_gu: Elaeis guineensis ; Eug_ma : Eugenia malaccensis ; Fic_be : Ficus benjamina ; Fic_ex : Ficus exasperata ; Hev_br : Hevea basiliensis; Lag_sp: Lagerstroemia speciosa; Man_in: Mangifera indica; Pel_pt: Peltophorum pterocarpum ; Ter_ca : Terminalia catappa ; Ter_me : Terminalia mantaly

\section{DISCUSSION}

L'étude a permis de recenser 29 espèces dont 22 dans la seule commune du Plateau. Une première étude réalisée par Vroh et al. (2014) a révélé la présence d'une moyenne de 6 espèces végétales en alignement contre 32 dans les espaces parcs et jardins dans la même commune. La faible richesse floristique enregistrée sur les plantations d'alignement par Vroh et al. (2014) serait due au fait que l'étude n'a pas pris en compte la majorité des voies de la Commune. Dans le cadre de cette étude, nos inventaires ont porté sur 11 voies pour la seule commune du Plateau. Les plantations d'alignement sur les avenues et boulevards seraient moins diversifiées. Seulement le Boulevard des Martyrs, le Boulevard Angoulevant et le Boulevard Clozel disposent d'un nombre d'espèces qui varient de 10 à 16 espèces. La faible richesse floristique dans les plantations d'alignement a été déjà signalée par Bekkouch et al. (2001) ; El-Lakany (2001) et Dardour et al. (2013) dans les villes respectives d'Oujda et Saïdia au maroc, de Bija au Portugal et du Caire en Egypte. Dans la présente étude, la richesse aurait été encore plus faible si on avait tenu compte uniquement des 


\section{Kouadio et al. J. Appl. Biosci. 2016 Évaluation de la diversité et estimation de la biomasse des arbres}

d'alignement des communes du Plateau et de Cocody (Abidjan - Côte d'Ivoire

individus d'espèces plantés à l'origine par les services techniques des différentes mairies et ignoré les épiphytes. En effet, sur les individus d'arbres plantés le long des boulevards et avenues, des épiphytes strictes et accidentelles (11 espèces) poussent sur les branches ou dans les fissures des écorces augmentant ainsi la richesse floristique dans ces espaces. La présence de chauve-souris dans les arbres du plateau serait à l'origine de la colonisation de ceux-ci dans les cavités humides des troncs ou encore dans les cimes des arbres (Benzing, 1990; Niamien et al., 2009). Néanmoins, sur toutes les voies, excepté l'avenue $D_{33}$ ou la seule espèce de Peltophorum pterocarpum est plantée, les autres voies comptent un minimum de deux espèces en association. Cette association des espèces en plantation d'alignement permettrait d'éviter les problèmes phytosanitaires liés à l'utilisation d'une seule espèce (Bekkouch et al., 2011). Le choix de ces espèces issues de la familles botaniques des Moraceae et des Caesalpiniaceae s'expliquerait par le fait que la plupart des espèces de ces familles s'adaptent mieux aux conditions climatiques et édaphiques de la ville (Merimi \& Boukroute, 1996; Dardour et al., 2013). Parmi elles, certaines ont un fort taux d'ombrage. C'est d'ailleurs, ce qui pourrait expliquer la forte proportion des espèces des zones forestières $(51,72 \%)$ qui sont des arbres sempervirents pouvant procurer de l'ombrage en toute saison (Leblanc \& Malaisse, 1978). D'autres s'y trouvent pour leur fleur. C'est le cas des espèces exotiques de la famille des Légumineuses, qui représentent $41,37 \%$ de l'ensemble des arbres d'alignement. Toutes ces caractéristiques indiquent que l'on a opté plus pour le caractère esthétique et récréatif lors de la mise en place des arbres d'alignement des communes du Plateau et de Cocody. En effet, la place des arbres d'alignement en milieu urbain a été longtemps limitée à de simple décor avant d'évoluer au fil du temps (Clergeau, 2007). Ainsi, les services les mieux connus des espèces recensées en alignement dans les deux communes semblent être l'esthétique et la récréation. Ce constat a été déjà fait par Zerbe et al (2004) dans leur étude dans la ville de Berlin. Au total 540 individus

\section{REMERCIEMENTS}

Nous traduisons notre profonde gratitude et notre reconnaissance à l'endroit des Responsables KRA Kouadio, Doumbia Brahima et Bendé Judicaël de la direction des parcs et jardins du district d'Abidjan pour les informations qu'ils ont bien voulu nous communiqué à $\mathrm{DBH} \geq 5 \mathrm{~cm}$ ont été recensés sur l'ensemble des boulevards et avenues. En termes de densité, on note que celle-ci est plus forte sur les avenues que sur les boulevards. Cela s'expliquerait non seulement par le fait que la plupart des arbres sur les avenues restent encore bien conservés. Sur certains boulevards comme le boulevard de la république qui est le plus long $(1,69$ $\mathrm{km}$ ) de la commune du plateau, plusieurs de ces arbres ont été abattus et remplacés par de jeune Chrisalidocarpus lutescens ou encore Roystonea regia devant certains bâtiments réhabilités. Leur présence gênerait la circulation des engins roulants au cours de la réhabilitation. Cet arrachage des arbres d'alignement a été déjà signalé par Haeringer (1980). Selon le même auteur, le bilan de l'arbre de rue reste maigre en Côte d'Ivoire dans la mesure où les arrachages l'emportent en nombre sur les nouvelles plantations. La biomasse est plus élevée sur les avenues que sur les boulevards. Aussi, les stocks de carbones à l'hectare sont-ils plus élevés sur les avenues que sur les boulevards. Ces différences de distribution de la biomasse et du stock de carbone s'expliqueraient par la distribution de l'aire basale et de la densité à l'hectare. Les plus fortes valeurs de ces deux paramètres mesurées sur les avenues témoignent de la présence d'un nombre élevé d'individus à grand diamètres. Le potentiel de stockage de carbones des arbres d'avenues et de boulevards rend compte de la participation des arbres d'alignement de la commune du Plateau et de Cocody à la réduction du dioxyde de carbone $\left(\mathrm{CO}_{2}\right)$, l'un des gaz à effet de serre désigné comme la première cause du réchauffement climatique (GIEC, 2004). En Côte d'Ivoire, le ministère en charge de l'environnement l'estimait déjà en 2008 à une quantité de 1.230 .000 tonnes dans l'atmosphère. Cette quantité de $\mathrm{CO}_{2}$ était émise par un parc automobile fort de 320.000 véhicules (MINEF, 2010) qui depuis lors continue d'augmenter. C'est pourquoi, il convient d'assurer la protection des arbres d'alignement et mieux de continuer le planting afin que ce patrimoine arboré continue de jouer son rôle compensatoire dans les émissions du dioxyde de carbone.

sur les espaces verts d'Abidjan. Notre reconnaissance va également à l'endroit de Monsieur ASSi Yapo Jean, Technicien botaniste qui a contribué à l'identification des espèces végétales inventoriées. 


\section{Kouadio et al. J. Appl. Biosci. 2016 Évaluation de la diversité et estimation de la biomasse des arbres}

d'alignement des communes du Plateau et de Cocody (Abidjan - Côte d'Ivoire

\section{CONCLUSION ET RECOMMANDATIONS}

L'étude réalisée sur les arbres d'alignement des communes du Plateau et de Cocody indique que le patrimoine arborée compte 705 individus. C'est un patrimoine qui abrite un important nombre d'espèce parmi lesquelles les plus représentatives sont au nombre de quatre. La plupart des espèces proviennent d'une part des espèces à croissance rapide et branchage solide de la région Guinéo-Congolaise et d'autre part de plantes exotiques ornementales. La biomasse végétale sèche assez élevée démontre d'un fort potentiel de stockage du carbone. A travers ce potentiel de stockage de carbone, l'étude a permis de mettre en évidence le rôle joué par les arbres d'alignement dans l'amélioration de la qualité de

\section{RÉFÉRENCE BIBLIOGRAPHIQUE}

Aguaron E. and McPherson EG, 2012. Comparison of Methods for Estimating Carbon Dioxide Storage by Sacramento's Urban Forest. Urban Ecosystems and Social Dynamics Program, USDA Forest Service, 1731 Research Park Dr, Davis, CA 95618, USA 300pp

Akbari $H$, Huang J, Martien P, Rainer L, Rosenfeld A, Taha $H, 2001$. Cool surfaces and shade trees to reduce energy use and improve air quality in urban areas. Solar energy, urban Environment $70: 295-310$.

Aké-Assi L, 2001. Flore de la Côte d'lvoire 1, catalogue, systématique, biogéographie et écologie. Genève, Suisse : Conservatoire et Jardin Botanique de Genève ; Boisseria 57, 396 pp.

Aké-Assi L, 2002. Flore de la Côte d'lvoire 2, catalogue, systématique, biogéographie et écologie. Genève, Suisse : Conservatoire et Jardin Botanique de Genève ; Boisseria 58, $441 \mathrm{pp}$.

Aké-Assi E, 2002. Contribution à l'étude des plantes ornementales cultivées dans les régions d'Abidjan et de San-Pédro, en Côte d'Ivoire, Doctorat de 3ème Cycle, U.F.R. Biosciences, Université de Cocody-Abidjan, Volume 1, 291pp

Akionla MA, 2012. Diversité et fonctions des formations végétales dans la ville de Porto-Novo. Mémoire de DESS en Gestion de l'Environnement, Université de Parakou, Bénin, 83pp.

Arrif T, Blanc N, Clergeau P, 2011. Trame verte urbaine, un rapport Nature-Urbain en l'environnement urbain. Ce rôle pourrait constituer une argumentation solide pour le maintien des arbres d'alignement dans la ville d'Abidjan dans un contexte de changement climatique. A l'issu de l'étude, des recommandations sont nécessaires pour la sauvegarde des arbres d'alignement des communes de Cocody et Plateau et partant de la ville d'Abidjan. La direction des parcs et jardins du district d'Abidjan devrait renforcer la sensibilisation et l'éducation environnementale pour éviter les actes d'incivisme et de vandalisme des populations à l'égard des arbres d'alignement. Elle devrait aussi œuvrer à la création de plantations d'alignement pour toute nouvelle voie construite.

géographie et écologie, Cybergo, European Journal of Geographie $17: 5-18$.

Bekkouch I, Kouddane NE, Daroui EA, Boukroute A, Berrichi A, 2011. Inventaire des arbres d'alignement de la ville d'Oujda, Nature \& Technologie $05: 87-91$

Benzing DH, 1990. Vascular epiphytes. Cambridge University Press, Cambridge. Journal of tropical ecology XVII : $354 \mathrm{pp}$.

Brown S. and Lugo AE, 1992. Aboveground biomass estimates for tropical moist forests of the brazilian amazon. Interciercia 17 (1): 8-18.

Brown S, 1997. Estimating biomass and biomass changes of tropical forests: A primer. FAO Forestry Paper 134, Rome, Italy.

Brown S, Gillespie AJR, Lugo AE, 1989. Biomass estimation methods for tropical forests with applications to forest inventory data. Forest Science 35:881- 902.

Clergeau $P, 2007$. Une écologie du paysage urbain. Ed. Apogée Rennes, France 210pp

Dardour M, Daroui EA, Boukroute A, Kouddane NE, Abdelbasset B, 2013. Inventaire et état sanitaire des arbres d'alignement de la ville de Saïdia (Maroc oriental), Nature \& Technologie $10: 02-09$.

Dye C, 2008. Santé et vie urbaine. Science. 319 : 766769.

Eldin M, 1971. Le climat de la Côte d'Ivoire. In Le milieu naturel de Côte d'Ivoire. Mémoires ORSTOM Paris (France) $50:$ 73-108.

El-Lakany MH, 2001. La foresterie urbaine et périurbaine au Proche Orient. Une étude de 
cas sur le Caire. Archives documents de la FAO. La foresterie urbaine et périurbaine, $5 \mathrm{pp}$.

Escobedo F, Varela S, Zhao M, Wagner JE, Zipperer W, 2010. Analyzing the effi cacy of subtropicalurban forests in offsetting carbon emissions from cities. Environ Sci Policy13 (5):362-372

GIEC 2004. Good practice guidance for land use, landuse change an forestry. Hayama, Japon, intergovernmental Panel on climate Change National Greenhouse Gas Inventories Programme (2004).

GIEC 2006. Guide pour l'inventaire national des gaz à effet de serre ; agriculture, foresterie et autre usage des terres. Institute for Global Environnemental Strategies Japon, 4: 46 - 52.

Haeringer P, 1980. L'arbre dans la ville: Lecture sociale en quatre tableaux du couvert végétal dans la ville africaine. Cahier O.R.S.T.O.M., série. Scientifique. Hum., vol. XVII : 289-308.

Jim C. and Chen W, 2008. Assessing the ecosystem service of air pollutant removal by urban trees en Guangzhou (China). Journal of Environnemental Management 88: 665-676

Jo $H, 2002$. Impacts of urban greenspace on offsetting carbon emissions for middle Korea. Journal of Environmental Management 64 : 115-126.

Leblanc M. et Malaisse F, 1978. Lubumbashi, un écosystème urbain tropical, Centre International de Semiologie, Université National du Zaïre. 178 pp

Lessard G. and Boulfroy E, 2008. Les rôles de l'arbre en ville, Centre collégial de transfert de technologie en foresterie de Sainte-Fory (CERFO), Québec, 21 pp.

Malhi Y, Baker TM, Phillips OL, Almeida S, Alvarez E, Arroyo L, 2004. The above-ground coarse wood productivity of 104 neotropical forest plots. Global Change Biology;10:563-91.

Mangenot G, 1955. Etude sur les forêts des plaines et plateaux de la Côte d'Ivoire : étude éburnéenne. Dakar (Sénégal) I.F.A.N (4) : 556.

Mehdi L, Weber C, Di Pietro F, Selmi W, 2012. Évolution de la place du végétal dans la ville, de l'espace vert à la trame verte ", VertigO - la revue électronique en sciences de l'environnement (12) 2. 22pp.

Merimi J. and Boukroute A, 1996. Inventaire et état sanitaire des arbres d'alignement dans la ville
d'Oujda (Maroc oriental), Actes Inst. Agron. Vet., Maroc (16) 1: 41-47.

Millennium Ecosystem Assesment, 2005. Ecosystem Wealth and Human Well-being. Island Press. 135pp.

Miller JR, 2005. Conservation de la biodiversité et l'extinction de l'expérience. Tendances Ecologie. Evolution 20 : 430-434.

MINEF, 2010. Atelier sous régional sur les stratégies de réduction des gaz d'échappement des véhicules et autres engins motorises. Allocution de Monsieur le ministre de l'environnement, des eaux et forêts, 5pp

Niamien $\mathrm{M}$, Yaokokore-Beibro $\mathrm{H}$, Kone I, Yao S, N'Goran E, 2009. Données préliminaires sur les habitudes alimentaires des chauves-souris paillées, Eidolonhelvum (Kerr, 1972) (Chiroptera : Pteropodidae) de la commune d'Abidjan Plateau (Côte d'Ivoire) Agronomie Africaine 21 (3) : 215-330.

Nowak DJ. and Crane D, 2002. Carbon storage and sequestration by urban trees in the USA. Environmental Pollution 116: 381-389.

Nowak DJ, 1994. Atmospheric carbon dioxide reduction by Chicago's urban forest. In: McPherson, E.G., Nowak, D.J., Rowntree, R.A. (Eds.), Chicago's Urban Forest Ecosystem: Results of the Chicago Urban Forest Climate Project. USDA Forest Service General Technical Report NE-186, Radnor, PA, 300pp.

Nowak DJ, Hoehn RE, Crane DE, Stevens JC, Walton JT, 2006. Assessing Urban Forest Effects and Values: Minneapolis' Urban Forest. USDA Forest Service, Northeastern Resource Bulletin, NE-166pp.

Nowak DJ, Rowntree RA, McPherson EG, Sisinni SM, Kerkmann E, Stevens JC, 1996. Measuring and analyzing urban tree cover. Landscape and Urban Planning 36: 49 - 57

Rusterholz HP, 2003. Biodiversité en milieu urbain : Protection de la nature en milieu urbain et rôle des espaces verts affectés à un entretien extensif. Institut pour la protection de la nature, du paysage et de l'environnement, Paris, $24 \mathrm{pp}$.

Schneiders A, Van Daele T, Van Landuyt W, Van Reeth W, 2012. Biodiversity and ecosystem services: Complementary approaches for ecosystem management? Ecological Indicators $21: 123-133$ 
Selmi W, 2011. Espace verts publics, entre planification urbaine et attentes des citadins. Laboratoire Image ville Environnement, Strasbourg, 50p

Véron J, 2007. La moitié de la population mondiale vit en ville, Population \& Sociétés $435: 1-4$.

Vroh BTA, Tiébré MS, N'Guessan KE, 2014. Diversité végétale urbaine et estimation du stock de carbone : cas de la commune du Plateau Abidjan, Côte d'Ivoire, Afrique Science 10(3): 329-340

Wolff $A, 2005$. La problématique de l'environnement urbain vue par une écologue. Le cas de l'île de France.». In Ville et environnement, Paris: Ellipses édition, pp : 204 - 223.

Young RF, 2010. Managing municipal green space for ecosystem service. Urban forestry and urban greening 9: 313-321.

Zerbe S, Maurer U, Schmitz S, Sukopp H, 2004. Biodiversity in Berlin and its potential for nature conservatiom. Landscape and Urban Planning 62 (3) : 139-148. 